\title{
An optimal continuous type investment policy for the surplus in a risk model
}

\author{
Seung Kyoung Choi ${ }^{a}$, Eui Yong Lee ${ }^{1, a}$ \\ ${ }^{a}$ Department of Statistics, Sookmyung Women's University, Korea
}

\begin{abstract}
In this paper, we show that there exists an optimal investment policy for the surplus in a risk model, in which the surplus is continuously invested to other business at a constant rate $a>0$, whenever the level of the surplus exceeds a given threshold $V>0$. We assign, to the risk model, two costs, the penalty per unit time while the level of the surplus being under $V>0$ and the opportunity cost per unit time by keeping a unit amount of the surplus. After calculating the long-run average cost per unit time, we show that there exists an optimal investment rate $a^{*}>0$ which minimizes the long-run average cost per unit time, when the claim amount follows an exponential distribution.
\end{abstract}

Keywords: risk model, surplus process, continuous type investment policy, long-run average cost, optimal investment rate

\section{Introduction}

Cho et al. (2016) introduced a continuous time surplus process in a risk model which involves a continuous type investment. The surplus in the risk model linearly increases at a constant rate $c>0$ due to the incoming premium. Meanwhile, claims arrive according to a Poisson process of rate $\lambda>$ 0 and decrease the level of the surplus jump-wise by random amounts which are independent and identically distributed with distribution function $G$ of mean $\mu>0$. Whenever the level of the surplus exceeds a given threshold $V>0$, the investment of the surplus to other business is continuously made at a constant rate $a(0<a<c)$, until the surplus process goes below $V>0$. The investment starts again, if the level of the surplus goes over $V>0$.

It is assumed that $c$ is larger than $\lambda \mu$, the expected total amount of claims per unit time, however, $c-a$ is assumed to be less than $\lambda \mu$ to keep the surplus process from being infinitely large. Cho et al. (2016) obtained the stationary distribution of the surplus level by forming martingales from the surplus process and applying the optional sampling theorems to the martingales. They also obtained the moment generating function of the stationary distribution by establishing and solving an integrodifferential equation for the distribution function of the surplus level.

In this paper, we study an optimal investment policy for the surplus in the risk model introduced by Cho et al. (2016). After assigning, to the risk model, two costs which are the penalty per unit time while the level of the surplus being under $V>0$ and the opportunity cost per unit time by keeping a unit amount of the surplus, we show that there exists an optimal investment rate $a^{*}>0$ which minimizes the long-run average cost per unit time.

\footnotetext{
${ }^{1}$ Corresponding author: Department of Statistics, Sookmyung Women's University, Cheongpa-ro 47-gil 100, YongsanGu, Seoul 04310, Korea. E-mail: eylee@ sookmyung.ac.kr
}

Published 31 January 2018 / journal homepage: http://csam.or.kr

(c) 2018 The Korean Statistical Society, and Korean International Statistical Society. All rights reserved. 
The classical risk model has been studied by many authors, for examples, Gerber (1990), Dufresne and Gerber (1991), and Gerber and Shiu (1997), by assuming that a ruin occurs if the surplus becomes negative. They have studied the ruin probability of the surplus and some interesting characteristics, such as the time of ruin, the surplus before ruin and the deficit at ruin. The core result on the ruin probability is well summarized in Klugman et al. (2004). Dickson and Willmot (2005) calculated the density of the time of ruin by inverting its Laplace transform.

However, in all of the above works, the surplus process is assumed to stop if the ruin occurs. Cho et al. (2013) introduced a risk model where the surplus process continues to move even though the level of the surplus becomes negative and an investment of the surplus is made, by a fixed amount, to other business jump-wise and instantly, if the level of the surplus reaches a given level. They obtained the characteristic functions of the transient and stationary distributions of the surplus process.

Lim et al. (2016) studied an optimal investment policy in the risk model introduced by Cho et al. (2013). After assigning, to the risk model, the reward of the investment, the penalty of the surplus being short and the opportunity cost of keeping the surplus, they showed that there exists a unique amount of the surplus being invested, which minimizes the long-run average cost per unit time.

In Section 2, we review some interesting characteristics of the risk model obtained by Cho et al. (2016) which are needed for the optimization. In Section 3, we assign, to the risk model, two costs which are the penalty per unit time while the level of the surplus being under $V>0$ and the opportunity cost per unit time by keeping a unit amount of the surplus, and calculate the long-run average cost per unit time. In Section 4 , by assuming that the amount of each claim independently follows an exponential distribution of mean $\mu>0$, we show that there exists a unique value $a^{*}>0$ of the investment rate, which minimizes the long-run average cost per unit time.

\section{Interesting characteristics}

In this section, we summarize several interesting characteristics in Cho et al. (2016), which are necessary to study the optimal investment policy. Cho et al. (2016) decomposed $\{U(t), t \geq 0\}$ into two processes $\left\{U_{1}(t), t \geq 0\right\}$ and $\left\{U_{2}(t), t \geq 0\right\}$. $U_{1}(t)$ is formed by separating the periods where $U(t) \geq V$ from the original process and connecting them together. $U_{2}(t)$ is similarly formed by separating the periods where $U(t) \leq V$ from the original process and connecting them together.

We, first, summarize the interesting characteristics of $\left\{U_{1}(t), t \geq 0\right\}$. The detailed proofs of the following propositions are given in Cho et al. (2016).

Proposition 1. Let $T_{1}$ denote the length of a cycle between two successive regeneration points where $U_{1}(t)=V$, then

$$
E\left(T_{1}\right)=\frac{E\left(Y^{2}\right)}{2 \mu[\lambda \mu-(c-a)]},
$$

where $Y$ denotes the claim amount following distribution function $G$.

Proposition 2. Let $P_{x}^{u}$ be the probability that $U_{1}(t)$, starting from $u(V \leq u \leq x)$, reaches $x \geq V$ before it goes below $V$, and let $P_{V}^{u}$ be the probability that $U_{1}(t)$ goes below $V$ without reaching $x \geq V$, then

$$
P_{x}^{u}=\frac{e^{\theta u}-e^{\theta V} M_{Y_{e}}(-\theta)}{e^{\theta x}-e^{\theta V} M_{Y_{e}}(-\theta)}=1-P_{V}^{u},
$$


where $M_{Y_{e}}(-\theta)=\{1 /(\mu \theta)\}\left[1-M_{Y}(-\theta)\right], M_{Y}(r)=\int_{0}^{\infty} e^{r y} d G(y)$, and $\theta>0$ is the solution of

$$
d(r)=r(c-a)+\lambda\left\{M_{Y}(-r)-1\right\}=0 .
$$

Proposition 3. Let $F_{1}(x)$ be the stationary distribution function of $U_{1}(t)$, then

$$
F_{1}(x)=1-\frac{P_{x}^{V}}{P_{V}^{\left(x-Y_{e}\right) \vee V}}, \quad \text { for } V \leq x<\infty,
$$

where $P_{V}^{\left(x-Y_{e}\right) \vee V}=1-G_{e}(x-V)+\int_{0}^{x-V} P_{V}^{x-y} d G_{e}(y)$ with $G_{e}$ being the equilibrium distribution function of $G, Y_{e}$ is the random variable having $G_{e}$ as its distribution function, and $a \vee b$ denotes the larger one of $a$ and $b$.

Remark 1. In the earlier analysis of Cho et al. (2016), $F_{1}(x)$ was calculated as

$$
F_{1}(x)=1-\frac{P_{x}^{V}}{P_{V}^{x-Y_{e}}}, \quad \text { for } V \leq x<\infty,
$$

which is wrong, however, since $F_{1}(V) \neq 0$ in this formula. Observe that $U_{1}(t) \geq V$, almost everywhere, except the points where $U_{1}(t)$ goes below $V$ due to the claims.

We, now, summarize the interesting characteristics of $\left\{U_{2}(t), t \geq 0\right\}$. The detailed proofs of the following propositions are given in Cho et al. (2016).

Proposition 4. Let $T_{2}$ denote the length of a cycle between two successive regeneration points where $U_{2}(t)=V$, then

$$
E\left(T_{2}\right)=\frac{E\left(Y^{2}\right)}{2 \mu(c-\lambda \mu)} .
$$

Proposition 5. Let $M_{2}(r)$ be the moment generating function of the stationary distribution function $F_{2}(x)$ of $U_{2}(t)$, then

$$
M_{2}(r)=\frac{2 e^{r V}(c-\lambda \mu)\left[\mu r-1+M_{Y}(-r)\right]}{r E\left(Y^{2}\right)\left[c r-\lambda+\lambda M_{Y}(-r)\right]} .
$$

\section{Long-run average cost}

In this section, we assign the following two costs to the risk model:

(i) $b$ is the penalty per unit time while the surplus is under level $V$. $V$ may be considered as the required level of the surplus for payments by the government.

(ii) $h$ is the opportunity cost incurred by keeping a unit amount of the surplus per unit time without using (investing) it.

These two are typical costs when we manage the surplus in a risk model. The first one increases as the investment rate $a$ increases, meanwhile, the second one decreases as $a$ increases. With these two costs being assigned to the risk model, we will study whether there exists an optimal investment rate a. 
By making use of the propositions in Section 2, we can calculate the long-run average cost per unit time. To do that, we, first, obtain the long-run average level of the original surplus process $\{U(t), t \geq 0\}$.

Let $E(U)=\lim _{t \rightarrow \infty} E[U(t)]$ the stationary expectation of $\{U(t), t \geq 0\}$. Observe that the original process, $U(t)$, is also a regenerative process with cycles in which the cycles of $U_{1}(t)$ and $U_{2}(t)$ alternate with weights $E\left(T_{1}\right)$ and $E\left(T_{2}\right)$, which are the expected lengths of cycles of $U_{1}(t)$ and $U_{2}(t)$, respectively. Hence, from the renewal reward theorem of Ross (1996, pp. 133-135), the stationary expectation of $U(t)$ is given by

$$
E[U]=\frac{E\left(T_{1}\right) E\left(U_{1}\right)+E\left(T_{2}\right) E\left(U_{2}\right)}{E\left(T_{1}\right)+E\left(T_{2}\right)},
$$

where $E\left(U_{1}\right)$ and $E\left(U_{2}\right)$ are the stationary expectations of $\left\{U_{1}(t), t \geq 0\right\}$ and $\left\{U_{2}(t), t \geq 0\right\}$, respectively.

$E\left(U_{1}\right)$ can be obtained from Proposition 3 in Section 2, which is

$$
E\left(U_{1}\right)=\int_{V}^{\infty} x d F_{1}(x)
$$

In the next section, we will calculate $E\left(U_{1}\right)$, when $G$ is an exponential distribution of mean $\mu$. $E\left(U_{2}\right)$ can be obtained from Proposition 5 in Section 2, which is

$$
E\left(U_{2}\right)=\left.\frac{d}{d r} M_{2}(r)\right|_{r=0} .
$$

In the next section, we will calculate $E\left(U_{2}\right)$, when $G$ is an exponential distribution of mean $\mu$.

Finally, noting that $T_{1}+T_{2}$ forms a regeneration cycle of $\{U(t), t \geq 0\}$, we can show, from the renewal reward theorem of Ross (1996, pp. 133-135), that the long-run average cost per unit time, as a function of the investment rate $a$, is given by

$$
C(a)=\frac{b E\left(T_{2}\right)}{E\left(T_{1}\right)+E\left(T_{2}\right)}+h E(U), \quad \text { for } c-\lambda \mu<a<c .
$$

Recall that the investment rate $a$ is assumed to be less than the premium rate $c$ and that $c-a$ is assumed to be less than $\lambda \mu$, the expected total amount of claims per unit time, to keep the surplus process from being infinitely large.

If the distribution function $G$ of the claim amount is given, we may obtain $C(a)$ in a more closed form, and hence, we can find, numerically, the optimal investment rate $a$, even though the formula of $C(a)$ may be complicate. If $G$ is an exponential distribution, however, we can find the explicit formula of the optimal investment rate $a$ which minimizes $C(a)$.

\section{An optimal investment policy}

In this section, we assume that the amount $Y$ of each claim, independently, follows an exponential distribution of mean $\mu>0$, and show that there exists an optimal investment rate $a^{*}>0$ which minimizes the long-run average cost per unit time.

We, first, calculate $F_{1}(x)$ and $E\left(U_{1}\right)$. Since $M_{Y}(r)=1 /(1-\mu r)$, for $r<1 / \mu$, the unique solution of $d(r)=0$ in Proposition 2 is given by

$$
\theta=\frac{\lambda \mu-(c-a)}{(c-a) \mu}>0
$$


Moreover, $M_{Y_{e}}(-\theta)=(c-a) / \lambda \mu$, and hence, $P_{x}^{u}$ and $P_{V}^{u}$, in Proposition 2, are

$$
P_{x}^{u}=\frac{e^{\theta u}+\mu \theta e^{\theta u}-e^{\theta V}}{e^{\theta x}+\mu \theta e^{\theta x}-e^{\theta V}}=1-P_{V}^{u}, \quad \text { for } V \leq u \leq x .
$$

To obtain $F_{1}(x)$ in Proposition 3, we need to find $P_{x}^{V}$ and $P_{V}^{\left(x-Y_{e}\right) \vee V}$. From the above equation,

$$
P_{x}^{V}=\frac{\mu \theta e^{\theta V}}{e^{\theta x}+\mu \theta e^{\theta x}-e^{\theta V}}
$$

and from Proposition 3,

$$
P_{V}^{\left(x-Y_{e}\right) \vee V}=1-G_{e}(x-V)+\int_{0}^{x-V} P_{V}^{x-y} d G_{e}(y),
$$

where $G_{e}(y)=1-e^{-y / \mu}$, since $G$ is the exponential distribution with mean $\mu$, and

$$
P_{V}^{x-y}=\frac{(1+\mu \theta)\left(e^{\theta x}-e^{\theta(x-y)}\right)}{e^{\theta x}+\mu \theta e^{\theta x}-e^{\theta V}} .
$$

After tedious calculation, we can show that

$$
P_{V}^{\left(x-Y_{e}\right) \vee V}=\frac{\mu \theta e^{\theta x}}{e^{\theta x}+\mu \theta e^{\theta x}-e^{\theta V}}
$$

Hence, from Proposition $3, F_{1}(x)$ is given by

$$
F_{1}(x)=1-e^{-\theta(x-V)}, \quad \text { for } x \geq V,
$$

which turns out to be a shifted exponential distribution. Therefore, $E\left(U_{1}\right)$ is

$$
E\left(U_{1}\right)=V+\frac{1}{\theta}=V+\frac{(c-a) \mu}{\lambda \mu-(c-a)} .
$$

Now, to obtain $E\left(U_{2}\right)$, we differentiate $M_{2}(r)$, in Proposition 5, once with respect to $r$ and let $r \rightarrow 0$. By applying the L'Hôspital's rule twice, we have

$$
E\left(U_{2}\right)=\frac{(c-\lambda \mu)\left[2 V E(Y)-E\left(Y^{2}\right)\right]-\lambda E(Y) E\left(Y^{2}\right)}{2(c-\lambda \mu) E(Y)} .
$$

Since $Y$ is an exponential random variable with mean $\mu, E(Y)=\mu$, and $E\left(Y^{2}\right)=2 \mu^{2}$, and hence, $E\left(U_{2}\right)$ becomes

$$
E\left(U_{2}\right)=V-\frac{c \mu}{c-\lambda \mu}
$$

$E\left(T_{1}\right)$ in Proposition 1 and $E\left(T_{2}\right)$ in Proposition 4 are, now, respectively,

$$
E\left(T_{1}\right)=\frac{\mu}{\lambda \mu-(c-a)} \quad \text { and } \quad E\left(T_{2}\right)=\frac{\mu}{c-\lambda \mu} .
$$


Therefore, $E(U)$, in Section 3, is given by

$$
E[U]=V+\frac{\mu\left[c(c-a)-\lambda^{2} \mu^{2}\right]}{(c-\lambda \mu)[\lambda \mu-(c-a)]} .
$$

Finally, the long-run average cost per unit time $C(a)$, in Section 3, becomes

$$
C(a)=\frac{b[\lambda \mu-(c-a)]}{a}+h E(U), \quad \text { for } c-\lambda \mu<a<c .
$$
$C(a)$

We, now, show that there exists a unique value $a^{*}>0$ of the investment rate, which minimizes

Theorem 1. If $b(c-\lambda \mu)>h c^{2} / \lambda, C(a)$ is minimized at

$$
a^{*}=\frac{b(c-\lambda \mu)^{2}+\sqrt{b h \lambda \mu^{2}(c-\lambda \mu)^{3}}}{b(c-\lambda \mu)-h \lambda \mu^{2}},
$$

otherwise, $C(a)$ is minimized at $a^{*}=c$.

Proof: Differentiating $C(a)$ with respect to $a$ gives

$$
C^{\prime}(a)=\frac{b(c-\lambda \mu)}{a^{2}}-\frac{h \lambda \mu^{2}}{[\lambda \mu-(c-a)]^{2}} .
$$

Reducing two fractions to a common denominator, we have

$$
C^{\prime}(a)=\frac{\left[b(c-\lambda \mu)-h \lambda \mu^{2}\right] a^{2}-2 b(c-\lambda \mu)^{2} a+b(c-\lambda \mu)^{3}}{a^{2}[\lambda \mu-(c-a)]^{2}} .
$$

Let $N(a)$ denote the numerator of $C^{\prime}(a)$. Observe that $N(a)$ is a quadratic function and $N(a)=0$ has two real valued solutions which are

$$
a_{1}=\frac{b(c-\lambda \mu)^{2}+\sqrt{b h \lambda \mu^{2}(c-\lambda \mu)^{3}}}{b(c-\lambda \mu)-h \lambda \mu^{2}} \quad \text { and } \quad a_{2}=\frac{b(c-\lambda \mu)^{2}-\sqrt{b h \lambda \mu^{2}(c-\lambda \mu)^{3}}}{b(c-\lambda \mu)-h \lambda \mu^{2}} .
$$

Note that $a_{1}$ is positive if and only if the coefficient of the quadratic term, $b(c-\lambda \mu)-h \lambda \mu^{2}$, say $Q$, is positive and that $a_{2}$ is always positive regardless of the sign of $Q$. Moreover, observe that

$$
\begin{aligned}
N(0) & =b(c-\lambda \mu)^{3}>0, \\
N(c-\lambda \mu) & =-h \lambda \mu^{2}(c-\lambda \mu)^{2}<0, \\
N(c) & =\left[b \lambda(c-\lambda \mu)-h c^{2}\right] \lambda \mu^{2} .
\end{aligned}
$$

Therefore, we may consider the following four exclusive cases:

(i) When $b(c-\lambda \mu)>h c^{2} / \lambda$,

$N(c)>0$ and the coefficient of the quadratic term $Q>0$ (since $c>\lambda \mu)$, hence, $0<a_{2}<c-\lambda \mu<$ $a_{1}<c$ and $N(a)$ is strictly increasing in $c-\lambda \mu<a<c$, that is, $C(a)$ is minimized at $a=a_{1}$. 
(ii) When $h \lambda \mu^{2}<b(c-\lambda \mu) \leq h c^{2} / \lambda$,

$N(c) \leq 0$ and $Q>0$, hence, $0<a_{2}<c-\lambda \mu<c \leq a_{1}$ and $N(a) \leq 0$ in $c-\lambda \mu<a<c$, that is, $C(a)$ is minimized at $a=c$.

(iii) When $b(c-\lambda \mu)=h \lambda \mu^{2}$,

$N(c)<0$ and $Q=0$, hence, $N(a)$ is linearly decreasing and $N(a)<0$ in $c-\lambda \mu<a<c$, that is, $C(a)$ is minimized at $a=c$.

(iv) When $b(c-\lambda \mu)<h \lambda \mu^{2}$,

$N(c)<0$ and $Q<0$, hence, $a_{1}<0<a_{2}<c-\lambda \mu$ and $N(a)<0$ in $c-\lambda \mu<a<c$, that is, $C(a)$ is minimized at $a=c$.

In summary, when $b(c-\lambda \mu)>h c^{2} / \lambda, C(a)$ is minimized at $a=a_{1}$, otherwise, $C(a)$ is minimized at $a=c$.

\section{Acknowledgements}

This research was supported by the Sookmyung Women's University Research Grants 2015.

\section{References}

Cho EY, Choi SK, and Lee EY (2013). Transient and stationary analyses of the surplus in a risk model, Communications for Statistical Applications and Methods, 20, 475-480.

Cho YH, Choi SK, and Lee EY (2016). Stationary distribution of the surplus process in a risk model with a continuous type investment, Communications for Statistical Applications and Methods, 23, 423-432.

Dickson DCM and Willmot GE (2005). The density of the time to ruin in the classical Poisson risk model, ASTIN Bulletin, 35, 45-60.

Dufresne F and Gerber HU (1991). Risk theory for the compound Poisson process that is perturbed by diffusion, Insurance: Mathematics and Economics, 10, 51-59.

Gerber HU (1990). When does the surplus reach a given target?, Insurance: Mathematics and Economics, 9, 115-119.

Gerber HU and Shiu ESW (1997). The joint distribution of the time of ruin, the surplus immediately before ruin, and the deficit at ruin, Insurance: Mathematics and Economics, 21, 129-137.

Klugman SA, Panjer HH, and Willmot GE (2004). Loss Models: From Data to Decisions (2nd ed), John Wiley \& Sons, Hoboken, NJ.

Lim SE, Choi SK, and Lee EY (2016). An optimal management policy for the surplus process with investments, The Korean Journal of Applied Statistics, 29, 1165-1172.

Ross SM (1996). Stochastic Processes (2nd ed), John Wiley \& Sons, New York. 